\title{
Human-Robot Interaction Based GUI
}

\author{
Rahmat Fauzi Siregar ${ }^{* 1}$, Ramadoni Syahputra ${ }^{2}$, Muhamad Yusvin Mustar ${ }^{3}$ \\ 1,2,3 Departement of Electrical Engineering, Faculty of Engineering \\ Universitas Muhammadiyah Yogyakarta, \\ Kampus Terpadu UMY, Jl. Lingkar Selatan, Kasihan Bantul, Yogyakarta 55183, Indonesia \\ e-mail: ${ }^{1}$ rfauzisiregar@gmail.com, ${ }^{2}$ ramadoni@umy.ac.id, ${ }^{3}$ yusvin@umy.ac.id
}

\begin{abstract}
Nowadays, technological developments interactions in general, couldn't be separated from the rapid development of computer technology and human interaction. So that, supporting the user to be able to develop various models of interactions, especially the interactions between humans and robots. This study proposes a model of design and manufacture of human and robot interaction, in the form of a visual display on a computer controlling the robot motion. Object interaction in this study is using 4WD Smart Car Robot. Some of the tools used to build a robot control system at the time of interaction, including Netbeans IDE, based on JAVA and Arduino. The control algorithm is applied to build an optimal control system to model an interaction between humans and robots. Model of human interaction and robot is fully carried by the user in control of robot motion. The proposed interaction model can be implemented in real terms, in designing and making a model of human interaction and robot.
\end{abstract}

Keywords: Human-Robot Interaction, JAVA, GUI, Arduino, Radio Telemetry

\section{Introduction}

Human-Robot interaction allows the exchange of information between human users with various types of machinery, computers and other electronic devices. Interaction with modern devices is most commonly done through visual, auditory or tactile user interface [1].

Software testing in general and Graphical User Interface (GUI) in particular is one of the major challenges in the life cycle of any software system. GUI testing is inherently more difficult than on a traditional interface testing (remote control) and the command - line. Some of the factors that make GUI testing different from traditional software testing and significantly more difficult is the large number of objects, a different look and feel of objects, many parameters associated with each object, the disclosure progressive, input the complex from a variety of sources, and output graphics , Testing techniques existing ones need to be adjusted/improved for the GUI, and new testing techniques are desirable aims to create something that is more efficient and effective [2].

Manuscript received February 2017, revised March 2017
GUI provides an easier way to use the various functions of the application by organizing into hierarchical forms of selection and presents only sensible choice appropriate to the context. Graphical User Interface aims to set the standard user actions and paradigms work to various components are presented graphically to users to the various forms of use and application context. GUI improve the usability of the application significantly, but also makes application development, testing and maintenance are significantly more difficult [2].

The interaction between human and robot requires communication between robots and humans, communication between robots and humans can be taken in several forms, but the forms are largely influenced by whether humans and robots are in the scope adjacent to each other or not, like most other technologies, robot requires the user interface to interact with people or humans. First, the paradigm of human interaction and robot consists only of humans acting on the machine, by simply pressing a button to complete a task [3].

Some of the tools used to build a robot control system at the time of the interaction, including the

Copyright $@ 2017$ Universitas Muhammadiyah Yogyakarta - All rights reserved 
NetBeans IDE, Arduino and Radio Telemetry. The control algorithm is applied to build an optimal control system to model an interaction between humans and robots. Model of human interaction and robot is fully carried by the user in control of robot motion. The proposed interaction model can be implemented in the real, in designing and making a model of human interaction and robot. Figure 1 shows the object interaction used in this study is the 4WD Smart Car Robot.

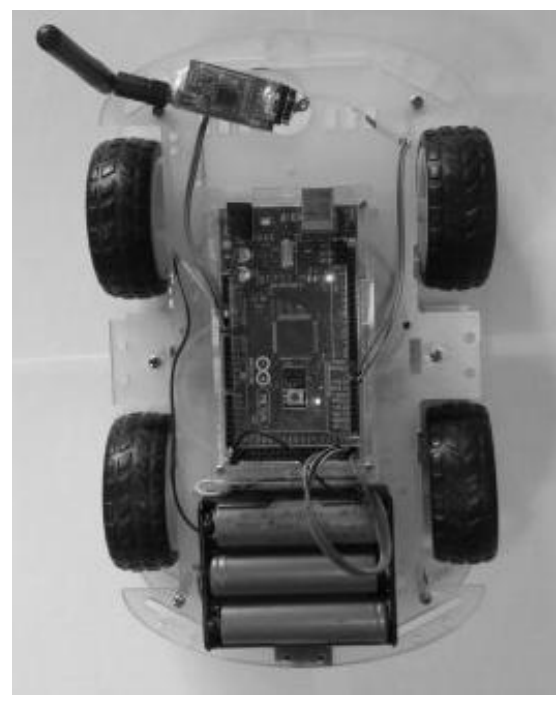

Fig. 1. 4WD Smart Car Robot

\section{Research Purposes}

Designing and implementing models of the interaction humans and robots in the form of GUI (Graphical User Interface) in controlling the movement of the robot.

\section{Theoretical Basis}

\section{III.1. Human-Robot Interaction}

The fundamental purpose of the interaction of humans and robots is to develop the principles and algorithms on a robotic system that allows it to interact directly with the safe and effective in humans, the interaction of humans and robots can be seen also as a communication process to an end user, the communication can be done in diverse modalities including voice dialogue, gestures, direct manipulation, and facial expressions. Interactions between humans and robots are defined, requiring communication between robots and humans, communication between humans and robots can be taken in several forms, but the forms are largely influenced by whether humans and robots are in the scope adjacent to each other or not [3].

Some paradigm [4], a theory [5] and taxonomy [6] of human-robot interaction has been described previously, it is very helpful in designing and developing various HRI models.

\section{III.2. Robot Control System}

The control system can be said to be a relationship between the components that make up a system configuration, which will generate the expected response of the system. So there must be controlled, which is a physical system, which is commonly called the plant. Basically, the control system consists of several basic components, namely the form of input, process control, and output. Input and output are variables or physical quantities. The output is produced by the plant, meaning controlled. Feedback is affecting the plant, which adjusts the output. Both dimensions of the input and output should not be the same. Control system used in the design of this system is an open control system [7].

\section{III.3. NetBeans IDE}

NetBeans is Integrated Development Environment (IDE) based on Java from Sun Microsystems that runs on top of Swing. Swing is a Java technology for the development of desktop applications that could be run on a variety of platforms such as Windows, Linux, Mac OS X and Solaris.

An IDE is the scope of programming integrated into a software application that provides builders Graphic User Interface (GUI), a text or code editor, a compiler or interpreter and a debugger. NetBeans is an Open Source software development, in other words, this software under development together and free of charge. NetBeans is an open source project that is successful with a very broad user community continues to grow and has nearly 100 partners. Sun Microsystems founded the NetBeans open source project in June 2000 and continues to be the main sponsor. Currently, there are two products: the NetBeans IDE and NetBeans Platform [8]. In this study, the NetBeans software is used as the controlling program, data processing and designing the movement of the robot visual models to interact with robots. 


\section{III.4. Arduino}

Arduino is known as physical or embedded computing platform, which means that interactive systems that can interact with its environment through the use of hardware and software. Arduino can sense the environment by receiving input from a variety of sensors and can affect its surroundings and is able to control a lamp, motors, actuators, and more. On the circuit, board microcontroller can be programmed using the Arduino programming language (based on Wiring) and the Arduino development environment (based on Processing). Arduino projects can be stand-alone or can communicate with software running on a computer [9].

Arduino Mega 2560 are used in this system. In addition, to having input and output pins are many, Arduino this type has a larger memory capacity compared to some other types of Arduino. To measure the dimensions of the device, include the type of Arduino Mega 2560 Arduino board with a large size.

\section{III.5. Library RXTXcomm}

The Java programming language also supports serial communications via the serial port. To be able to do the serial communication can use/exploit one of the libraries that RXTXcomm [10].

This library supports serial communication is applied to a Java-based program. This library can also be used on several platforms such as Linux, Solaris, and Windows.

\section{III.6. DC Motors}

DC motor is an electronic device. It can converts electrical energy into mechanical energy in the form of rotational motion. DC motors work on the principle of Lorentz force, which states that when a conductor which has a current flow is placed in a magnetic field, then a force (known as the Lorentz force) will be created orthogonally between the magnetic field direction and the direction of current flow [11]. In designing this system DC motor is used as a producer of the robot's movement.

\section{III.7. L298 Dual H-Bridge Driver}

IC L298 is Dual Full Bridge Driver IC operating voltages up to $46 \mathrm{~V}$ and $4 \mathrm{ADC}$ current, which is designed to accept standard TTL logic level. This IC serves to drive the induction load such as relays, solenoids, DC motors, and motors stepper [12].
In designing this system L298 Dual H-Bridge Driver is used as the control and direction of rotation of the DC motor speed.

\section{III.8. Data Communication}

Data communication is part of the communication that specifically addresses the transmission or transfer of data and information among computers and other devices in a digital form that is sent through the transmission medium. Data means the information presented by the digital cues. A data communication, there are several components, namely the data, transmitter, transmission medium, the receiver and the purpose [13].

In designing this system the transmission method used is the simplex method. The simplex method is one type of data communications that transmit information in only one direction.

\section{III.9. RC Timer Radio Telemetry Kit $433 \mathrm{MHz}$}

The device is made based radio telemetry 3DR Radio System and 100\% compatible. Open source telemetry as another option from the XBee. It can communicate from a distance at about 1 mile. The telemetry system uses full-duplex communication using HopeRF module HM - TRP that has been customized with open source firmware. Interface device uses a standard 5V TTL serial or USB FTDI Serial. To update and adjust the settings of the module can use the APM Mission Planner. Configuration can also be done with 3DR Radio Configurator or AT Command [14]. In designing this system RCTimer Radio Telemetry Kit 433 $\mathrm{MHz}$ is used as a medium for the transmission of data communications.

\section{Research Methodology}

\section{IV.1. Tools and Materials}

The tools used in the design of this system is a PC / Laptop with Windows 10 Pro 64 bit operating system, software NetBeans IDE version 8.2, libraries RXTXcomm, and the Arduino IDE software version 6.1.13, while the materials used are system design kit robot smart car 4 WD, RCTimer Radio Telemetry Kit $433 \mathrm{MHz}, \mathrm{L} 298$ Dual H-Bridge Driver, Battery 1800 mah Lipo 3S 20C and Arduino Mega 2560. 


\section{IV.2. System Planning}

This research was conducted by several stages, as shown in Figure 2 below.

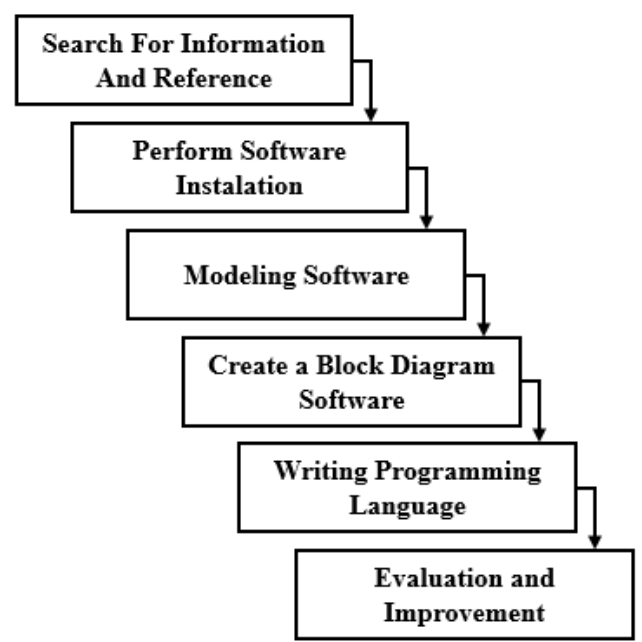

Fig. 2. Block diagram of the design stage models of human interaction and robot

This study used the waterfall model, which is aim to help overcome complexity caused by software development projects. This model allows the solution of complex development mission into some logical steps (requirements, specifications, design, code, unit test, testing, and maintenance) with some steps that will eventually become the final product is ready to use. To ensure that the system can be run, each step requires validation, input, and the existing criteria. Figure 3 shows a diagram of the method waterfall model.

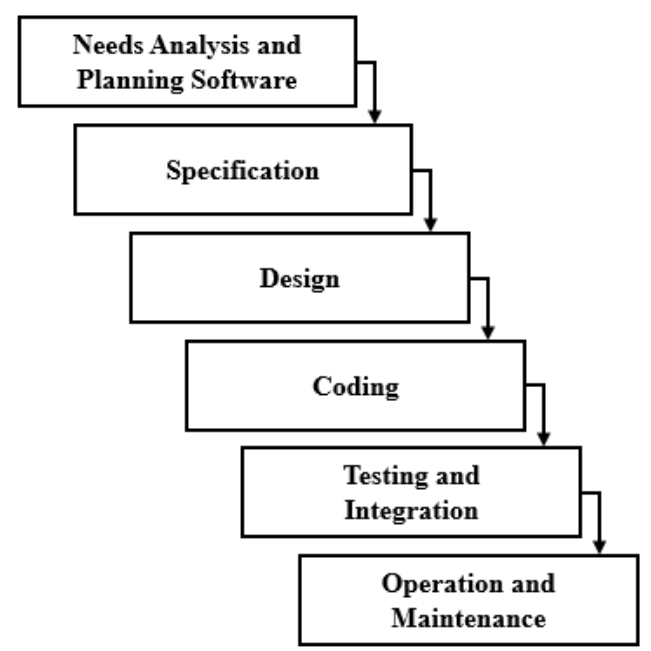

Fig. 3. Diagram of the method waterfall model

To meet user needs and satisfaction made an approach model that provides a prototype of a part or the whole system. A prototype is part of a product that expresses the logic and physical external interface that is displayed. Figure 4 shows a block diagram of a prototype approach in general.

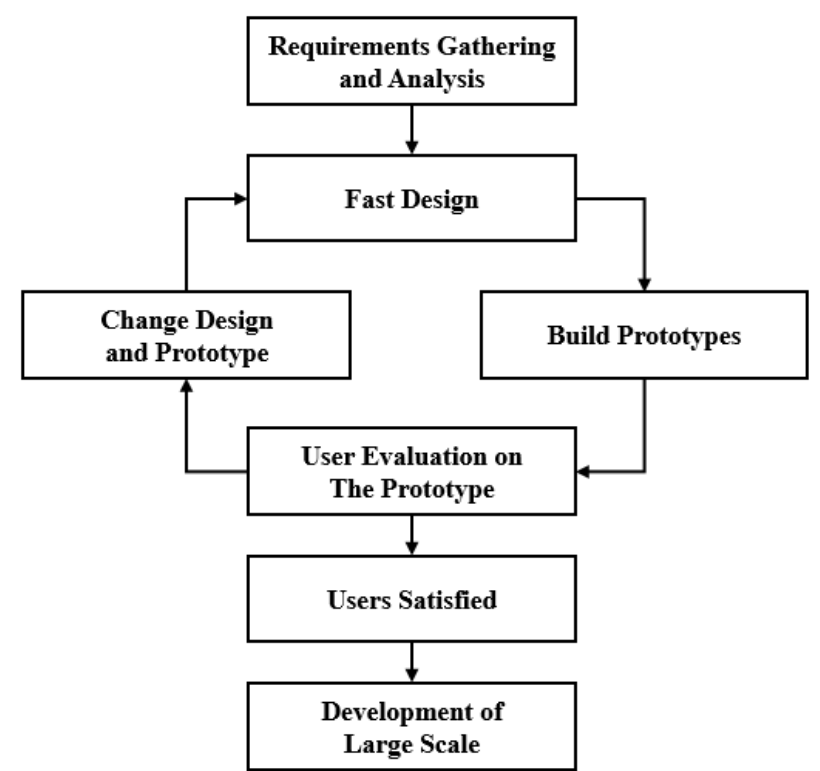

Fig. 4. Block diagram of a prototype approach in general

In this study, the component needed to build a GUI is a laptop, radio telemetry transmitter module and software NetBeans 8.2. GUI designed in NetBeans software by exploiting a Java application using the JFrame Form. JFrame Form is a GUI designed and design. GUI that works according to the research that is done takes some libraries such as JDK 1.8 (Default), absolute layout and RXTXcomm. Libraries JDK 1.8 (Default) and absolute layout functions to design the GUI while the library RXTXcomm function as serial communication. Figure 5 shows a block diagram of a GUI builder.

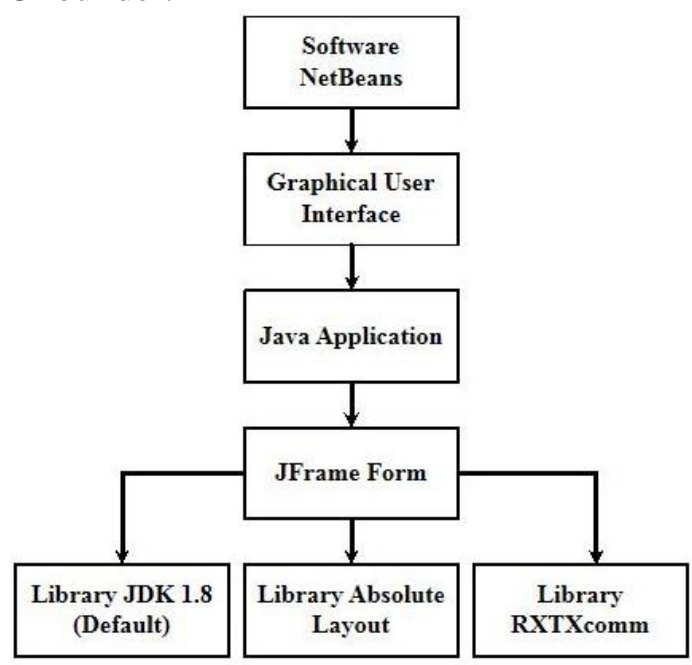

Fig. 5. A block diagram of a GUI builder 
In this study, the design of the system consists of receiver and transmitter. Components needed in this system design receiver is Arduino Mega 2560 to function as the control system, Battery $3 \mathrm{~S} 25 \mathrm{C}$ $1800 \mathrm{mAh}$ serves as a voltage source Arduino Mega 2560 and Driver L298N Dual H-Bridge Driver L298N Dual H-Bridge serves as the driving DC motors, DC motors function as the driving force of each wheel on the car kit smart, Radio Telemetry
$433 \mathrm{MHz}$ Receiver function as a data receiver. While the components needed in the design of the system transmitter is Radio Telemetry Transmitter $433 \mathrm{MHz}$ serves as the sender data and Computer serves as a means of controlling the robot via the GUI design that runs based on NetBeans software. Figure 6 shows a block diagram of the system.

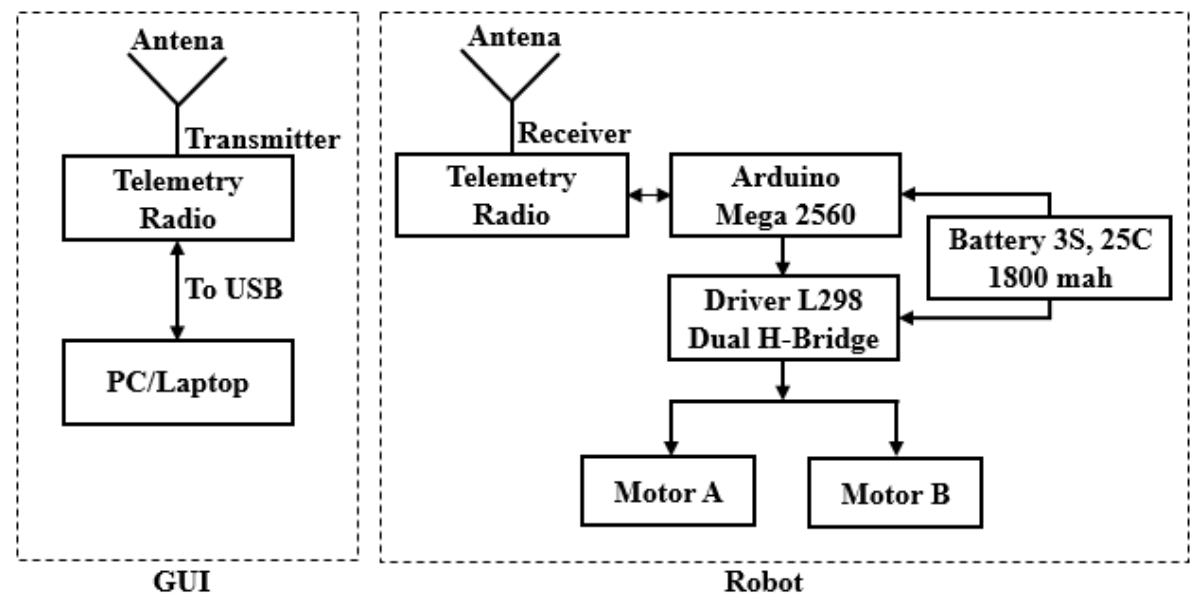

Figure 6. A block diagram of the system

\section{Result and Explanations}

\section{V.1. Research Result}

This research resulted in three pieces of design Graphical User Interface (GUI) that is a GUI version 1.0 , version 1.1 and version 1.2 and the design of robot smart car kit.

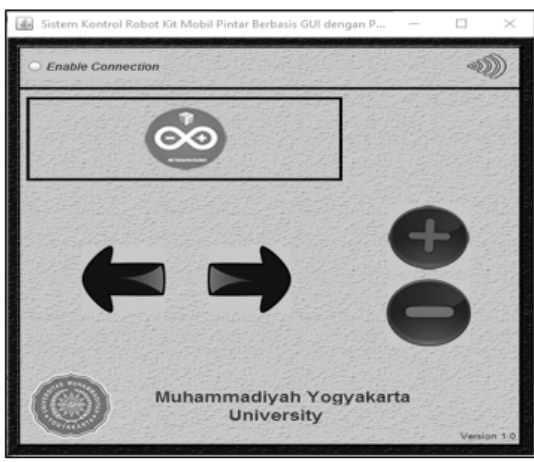

GUI version 1.0

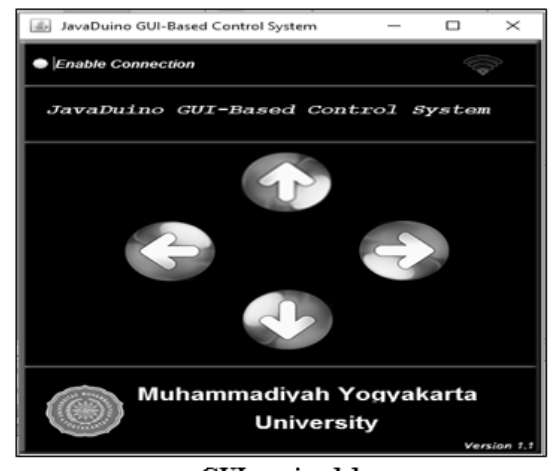

GUI version 1.1
Design Graphical User Interface was designed based on the method of the waterfall model, the best color combinations and color combinations ugliest. The combination of colors on a GUI design serves to avoid the eyes from fatigue, look more attractive, and easier for users to understand GUI. Figure 7 shows the results of GUI design

Fig. 7. The results of GUI design

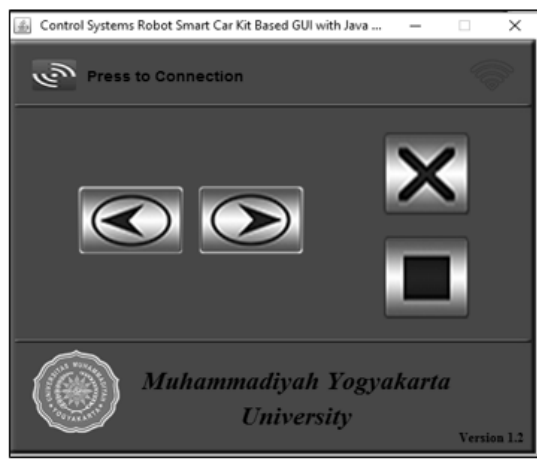

GUI version 1.2
GUI design used in the study is the design of the GUI version 1.2. This design was selected based on the results of the questionnaire are selected by some of the participant's questionnaires. In version 1.0
GUI design two people chose a very bad, five people choose bad, twelve people choose good and seven people chose very good. In version 1.1 the GUI design one people choose very badly, two 
people choose bad, eighteen people choose good and five people chose very good. In version 1.2 GUI design one person chose a very bad, four people choose badly, eleven people choose good and ten people chose very good. Figure 8 shows the results of questionnaires GUI design.
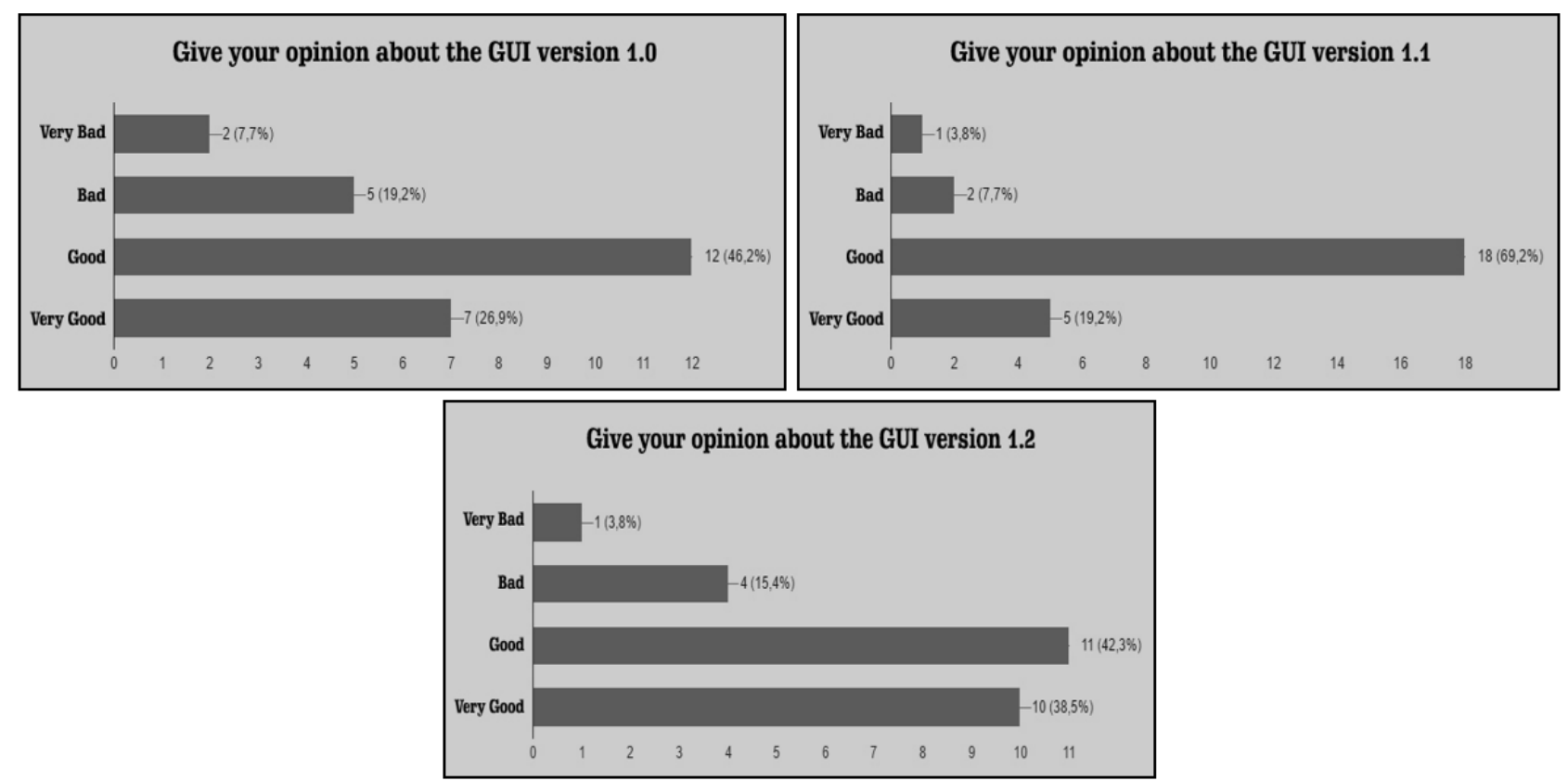

Figure 8 . The results of questionnaires GUI design

\section{V.2. Examination}

Tests conducted in the form of trials modeling the interaction with the robot, by connecting the serial communication by pressing button connection. Button connection used to connect GUI with Arduino via serial communication by utilizing radio module Telemetry Kit $433 \mathrm{MHz}$ as a medium. In the source code button, connection has variable name butwifi. In the class butwifi, there are some methods as shown in Figure 9 below.

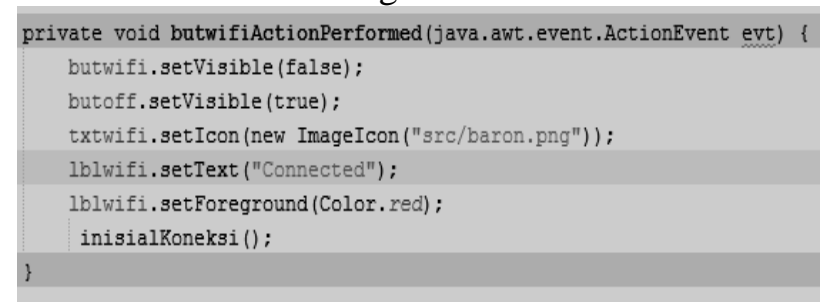

Fig. 9. Methods in the class butwifi

In order to give the action when pressing the button, the connection button is given event Performed action. At the time of the connection button on tap, all classes and methods in this connection button class will be executed.

In the class of inisialKoneksi (); is a serial communication can be accessed and this class that serves as the connecting COM 3 serial port and baud rate 57600 .

In the method butwifi.setVisible (false); states that the class butwifi can not be seen when the connection has been connected, it means the button connection will be lost when the connection has been connected. This button connection size is $27 \mathrm{x}$ 27 pixels.

In the method butoff.setVisible(true); states butoff class can be seen when the connection has been connected, it means the connection breaker button will appear when the connection has been connected. This connection breaker button size is $25 \times 25$ pixels.

In the method txtwifi.setIcon (new ImageIcon ("src / baron.png")); stated that a label with the name of the variable txtwifi will take a new icon with the name of the folder src baron with PNG format, meaning that when the connection has been connected icon wifi picture empty bar will change to icon wifi picture full bar. This picture icon size is 40 x 40 pixels.

In the method lblwifi.setText("Connected"); stated that a label with the name of the variable lblwifi will change the text which is basically "Press to Connection" to text "Connected" when the 
connection has been connected. Text size and font on the label is the Vani, Bold, 14 pt.

In the method lblwifi.setForeground (Color.red); states that the text color on this label, which is basically black will turn red when a connection has been connected.

Figure 10 shows before the button connection are pressed and after the button connection is pressed.

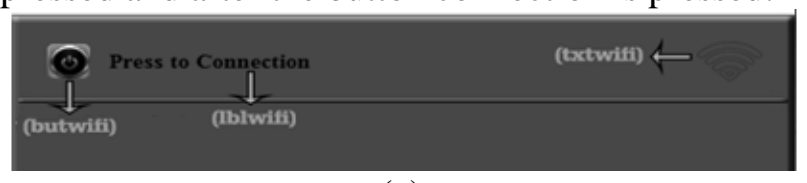

(a)

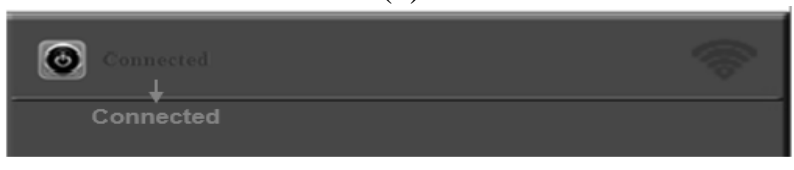

(b)

Fig. 10. Before the button Connection is pressed (a) and after the button connection is pressed (b)

Furthermore, the forward button testing. Forward button functions to control the robot move forward. In class the forward button with variable maju given event mousePressed. Event mousePressed used when the user presses the mouse. In the class forward (majuMousePressed) there are several methods as shown in Figure 11 below.

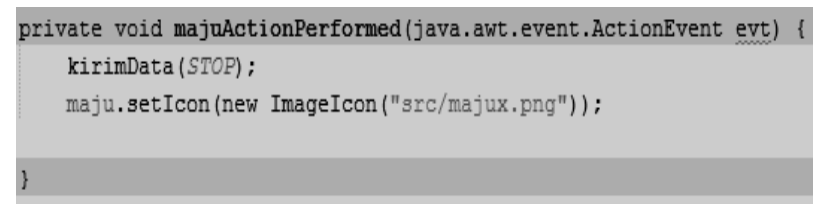

Fig. 11. Method in the class majuMousePressed

Both of method will be executed when the forward button is pressed with the mouse. To control the robot move forward, method kirimData (FORWARD); used to send data initialization character " $F$ " through the serial port on the laptop to an Arduino in robot with radio media Telemetry Kit. Method maju.setIcon (new ImageIcon ("src / majuon .png")); used to display the new image icon with the file name majuon contained in the src folder. These the forward button size is $133 \times 109$ pixels. Figure 12 shows the flowchart and working principle of the forward button.
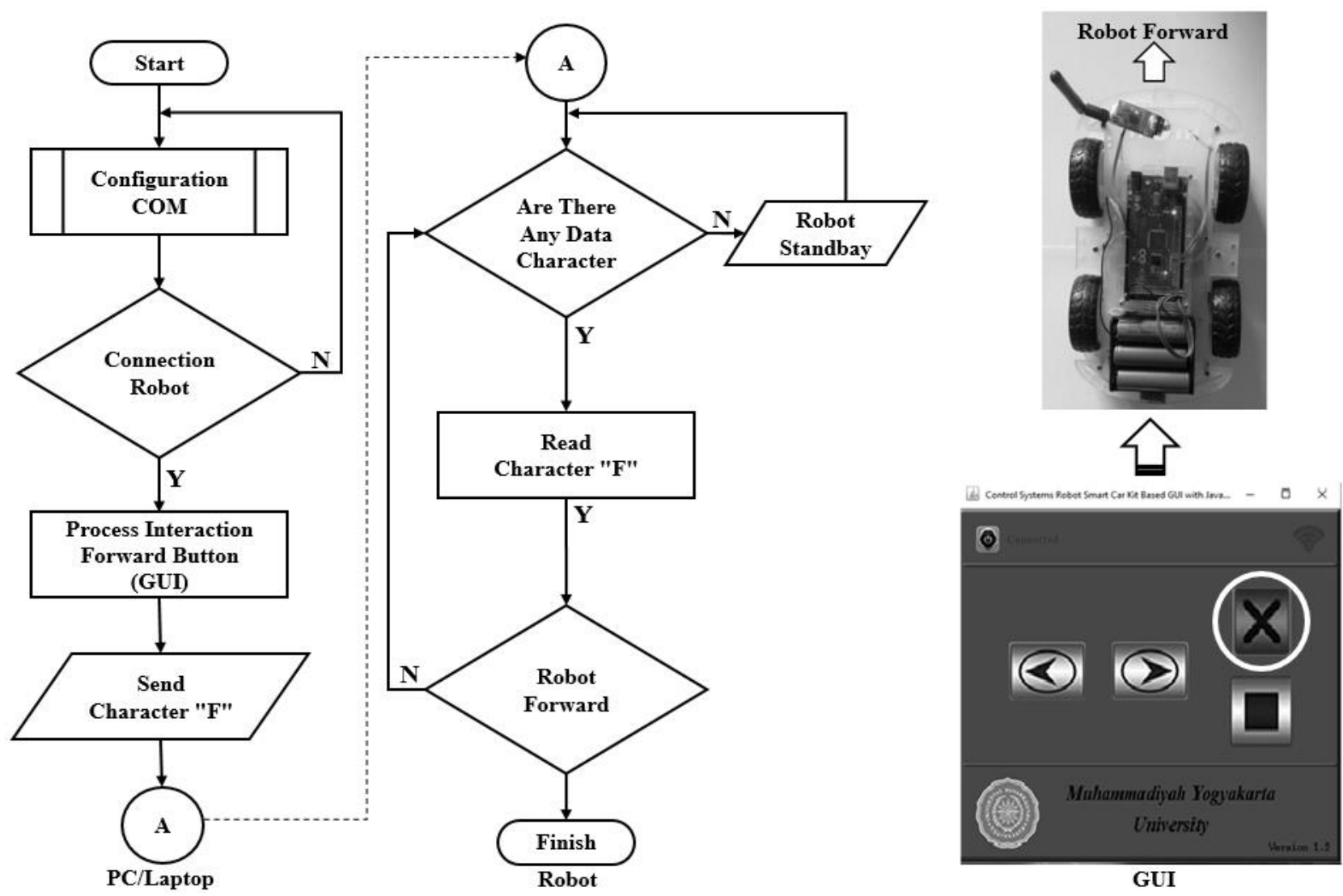

Fig. 12. The flowchart and working principle of the forward button 
As can be seen in Figure 12, flowchart and working principle of the forward button, have in common with the method of the backward button, turn right button and turn left button. The table 1 shows button function and character.

TABLE I

BUTTON FUNCTION AND CHARACTER

\begin{tabular}{|c|c|c|c|}
\hline Button & Button Size & Functions & Character \\
\hline & $\begin{array}{c}133 \times 109 \\
\text { pixels }\end{array}$ & $\begin{array}{c}\text { Robot } \\
\text { move } \\
\text { forward }\end{array}$ & "F" \\
\hline & $\begin{array}{c}133 \times 109 \\
\text { pixels }\end{array}$ & $\begin{array}{c}\text { Robot } \\
\text { move } \\
\text { backward }\end{array}$ & "B" \\
\hline & $\begin{array}{c}153 \times 89 \\
\text { pixels }\end{array}$ & $\begin{array}{l}\text { Robot } \\
\text { rotates to } \\
\text { the right }\end{array}$ & "R" \\
\hline & $\begin{array}{c}153 \times 89 \\
\text { pixels }\end{array}$ & $\begin{array}{l}\text { Robot } \\
\text { rotates to } \\
\text { the left }\end{array}$ & "L" \\
\hline
\end{tabular}

Furthermore, test the stop button. Stop button serves to control the robot to stop moving. Stop button class represented by the forward, backward, turn right and turn left with event actionPerformed. Event actionPerformed used when the event action occurs. In this stop button class there are method kirimData(STOP); and a method that is used to display a picture of each navigation buttons to the initial shape. Both of method will be executed when the entire navigation button is not pressed or one of the navigation buttons has been pressed and then released. Method kirimData(STOP); used to send data initialization character "S" through the serial port on the laptop to an Arduino in robot with radio media Telemetry Kit.

Furthermore, test the connection breaker button. Button connection breaker is used to disconnect the GUI with the Arduino via serial communication by utilizing radio module Telemetry Kit $433 \mathrm{MHz}$ as a medium. In the source code breaker button, the connection has butoff variable name. In class butoff there are several classes and method as shown in Figure 13 below.

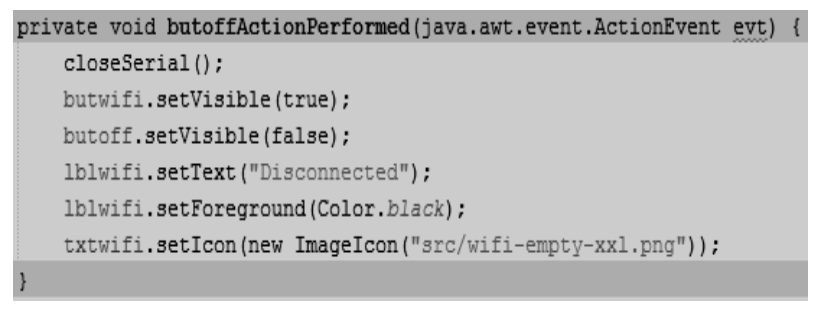

Fig. 13. Method and class in the class butoffActionPerformed
In order to give the action when pressing the button, the connection breakeris given action Performed event. At the time of connection, breaker button is pressed all classes and methods in the class this connection button will be executed.

In class closeSerial(); a class in charge of deciding the serial connection or can be called also by closing the serial port between GUI with Arduino and an assigned class window displays the message "Koneksi terputus!".

In butwifi.setVisible(true) method; states that the butwifi class can be seen when the connection has been disconnected, it means the connection button will appear when the connection has been disconnected. This connection button size is $27 \mathrm{x}$ 27 pixels.

Butoff.setVisible(false) method; states that the butoff class cannot be seen when the connection has been disconnected, which means that the breaker button the connection will be lost when the connection has been disconnected. This connection breaker button size is $25 \times 25$ pixels.

In method lblwifi.setText("Disconnected"); states that a label with the name of the variable lblwifi will change the text when the connection has been disconnected becomes "Disconnected". Text size and font on the label is Vani, Bold, 14 pt. In the method lblwifi.setForeground (Color.black); states that the text on the label color will change to black when the connection has been disconnected.

In the method txtwifi.setIcon(new ImageIcon ("src/wifi-empty-xxl.png")); states that a label with variable name txtwifi will take a new picture icon of the src folder with the name of wifi-empty picture-xxl with PNG format, meaning that when the connection has been disconnected icon image bar full wifi will turn into a icon wifi picture empty bar. This picture icon size is 40 x 40 pixels. Figure 14 shows the GUI display when the connection has been connected and after connection, breaker button is pressed.

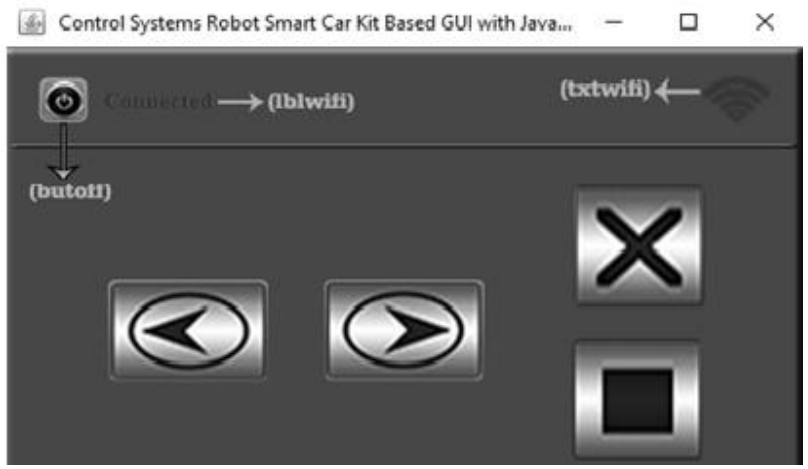

(a) 


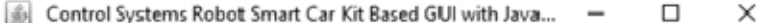

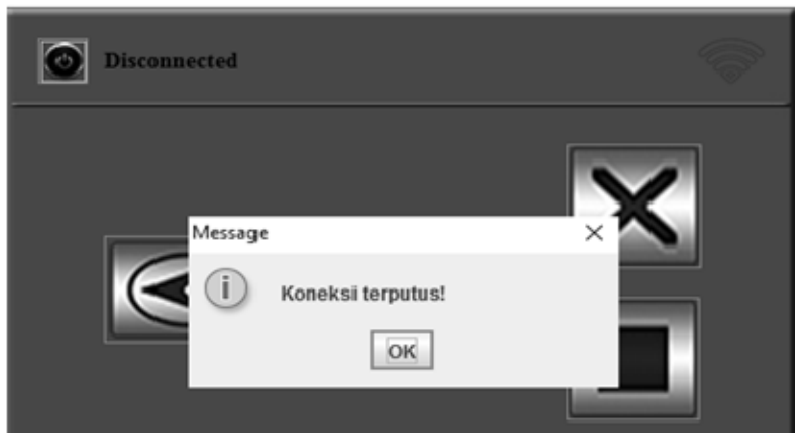

(b)

Fig. 14. GUI display when the connection has been connected (a) and after connection breaker button is pressed (b)

Last, testing the distance of the range of transmission between the GUI with the robot performed in Maguwoharjo Stadium. The distance can be reached in about 960 meters.

After the testing is done and the GUI runs well, then change the GUI into software that can run on Windows and Macintosh operating systems. To be can run on the Windows and Macintosh operating systems, becoming an installer GUI created using software Advanced Installer 13.5. This software named JavaDuino V1.2

\section{Conclusion}

Based on the experiment and result of questionnaires showed the GUI can be implemented as a model of human interaction and robot in controlling the movement of the robot. In designing the GUI requires some prototypes that can later become the basis of a GUI design that aims to meet the needs of the user. By using the approach to the method of the waterfall model can serve as the basis for designing software.

In the future work, GUI can be developed further by adding a variety of information such as a camera and various sensors, which is give impact to the human-robot interaction model interactive.

\section{Acknowledgements}

This work was supported by Universitas Muhammadiyah Yogyakarta.

\section{References}

[1] Sodnik, Jaka., dan Tomazic, Saso. (2015). Spatial Auditory Human-Computer Interfaces. Springer. 1-2.
[2] Singhera, Zafar., Horowitz, Ellis., and Shah, Abad. (2009). A Graphical User Interface (GUI) Testing Methodology. Chapter: 7.24, 1-3.

[3] Yusvin Mustar M. (2014). Media Interaksi ManusiaRobot Berbasis Sensor Accelerometer dan Flex (Khusus Gerak Tangan dan Lengan). M.Eng Thesis. Yogyakarta: Universitas Gadjah Mada.

[4] Breazeal C.(2004). Social interactions in HRI: the robot view. Syst Man Cybern Part C Appl Rev IEEE Trans On. 34(2):181-6.

[5] Scholtz J. (2003). Theory and evaluation of human robot interactions. System Sciences, Proceedings of the 36th Annual Hawaii International Conference on. IEEE

[6] Yanco HA, Drury JL. A. (2002) taxonomy for humanrobot interaction. Proceedings of the AAAI Fall Symposium on Human-Robot Interaction. p. 111-9.

[7] Hedwig, Rinda. (2004). Teori Sistem. Jakarta: Universitas Bina Nusantara, 1-12.

[8] NetBeans. "A Brief History Of NetBeans",25-Des2016. [Online]. Available: https://netbeans.org/about/history.html. [Accessed: 25-Des-2016].

[9] “Arduino Mega 2560 and Genuino Mega 2560 “ 25 Des-2016. [Online]. Available: https://www.arduino.cc/en/Main/ArduinoBoardMega 2560. [Accessed: 25-Des-206].

[10]Bock, Heiko. (2009). The Definitive Guide to NetBeans Platform. Academic PRESS. 1-14.

[11]Leondes, C. T. (1972). Control and Dynamic Systems. Academic PRESS, Inc. 39 (6), 1 - 34.

[12]“ Data Sheet L298 Dual Full - Bridge Driver “ 25 Des-2016. [Online]. Available: https://arduinoinfo.wikispaces.com/L298N-DataSheet. [Accessed: 25-Des-2016].

[13]Forouzan, Behrouz A. (2009). Data Communication and Networking. Kansas: The University of Kansas, (1), 6-17.

[14] "Data Sheet RCTimer Radio Telemetry Kit $433 \mathrm{MHz}$ " 25-Des-2016. [Online]. Available: http://rctimer.com/product-834.html. [Accessed: 25Des-2016].

\section{Authors' information}

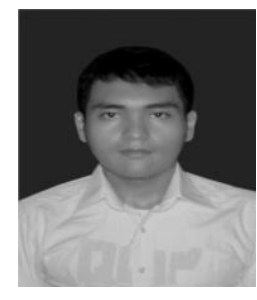

Rahmat Fauzi Siregar, born on 21 November 1994, Padangsidimpuan, North Sumatra. Received B.Sc. degree from Universitas Muhammadiyah Yogyakarta in 2017.

Rahmat Fauzi Siregar, S.T. His research interests are in software engineering, humanrobot interaction, microcontroller, humanmachine interaction, robotics, electrical and electronics engineering. 


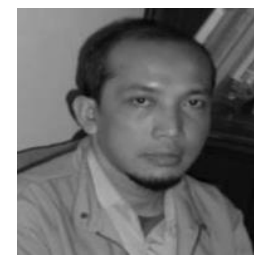

Surabaya, Indonesia.

Ramadoni Syahputra received B.Sc. degree from Institut Teknologi Medan in 1998, M.Eng. degree from Department of Electrical Engineering, Universitas Gadjah Mada, Yogyakarta, Indonesia in 2002, and Ph.D degree at the Department of Electrical Engineering, Faculty of Industrial Technology, Institut Teknologi Sepuluh Nopember,

Dr. Ramadoni Syahputra is a Lecturer in Department of Electrical Engineering, Faculty of Engineering, Universitas Muhammadiyah Yogyakarta, Indonesia. His research interests are in computational of power system, artificial intelligence in power system, power system control, the application of fuzzy logic in power system, optimization, distributed energy resources, and renewable energy.

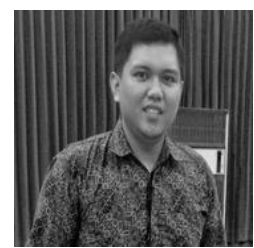

Muhamad Yusvin Mustar Received Diploma degree Electrical Engineer from Universitas Haluoleo, Kendari in 2009, B.Sc. degree from from Department of Electrical Engineering Universitas Muhammadiyah Yogyakarta in 2011, M.Eng. degree from Department of Electrical Engineering and Informatics Technology, Universitas Gadjah Mada, Yogyakarta, Indonesia in 2014.

Muhamad Yusvin Mustar, M.Eng. is a Lecturer in Department of Electrical Engineering, Faculty of Engineering, Universitas Muhammadiyah Yogyakarta, Indonesia. His research interests are in human-robot interaction, human-machine interaction, robotics, electrical and electronics engineering. 